\title{
Compound heterozygosity for a hemizygous rare missense variant (rs141999351) and a large CNV deletion affecting the FSTL5 gene in a patient with schizophrenia
}

Reports of associations between some large and rare copy number variations (CNVs) and schizophrenia have considerably enriched the debate on the genetics of schizophrenia. Although CNVs represent plausible risk factors, their presence in unaffected individuals is likely to implicate the involvement of additional genetics and/or environmental factors in the development of schizophrenia, as exemplified by the double-hit hypothesis. In the case of $\mathrm{CNV}$ deletions, the second hit might be, for instance, a point mutation unmasking a recessive allele.

This last possibility is sustained by data of a 58-year-old man with a DSM-IV-TR diagnosis of paranoid schizophrenia and no evidence of Axis I or II disorders. The patient was identified as a carrier of a large deletion during the CNV screening of a cohort of Italian schizophrenia patients using the SNP-array technology (Magri et al., 2010). Briefly, genotyping was achieved with the Affymetrix Human Mapping GeneChip 6.0 arrays, intensity data were analyzed using the BRLMM-P Plus algorithm and CNVs identified using the segmentation algorithm implemented in the Affymetrix Genotype Console (GTC) 3.0.1 (for more detailed information, see Magri et al. (2010)). This analysis identified in the patient an about 2,300 kb deletion on chromosome 4q32.1-32.2 (chr4:160,666,009-162,968,318 hg19 assembly). The deletion encompasses the FSTL5 (follistatin-like 5) gene, extending from intron 2 to approximately 1,640 kb downstream the 3 ' end of the gene and removing nearly all the coding sequence. As a result of this finding, we planned a mutational analysis of the FSTL5 gene in the patient. The Sanger sequencing of the 15 coding exons of the gene and their intronic flanking regions revealed a T > C transition at position 1358 of the cDNA (c.1358T > C NM_020116; chr4:162,421,268A > G hg19 assembly), resulting in a methionine to threonine substitution at position 453 (p.Met453Thr) of the amino acid sequence (NP_064501). This substitution was predicted to be "damaging” by SIFT (http://sift.jcvi.org) and “disease-causing” by MutationTaster (http://www.mutationtaster. org) servers.

We then screened 164 patients with schizophrenia and 246 control subjects for the c.1358T $>\mathrm{C}$ transition. The mutation was found in heterozygosity in a second patient and in one control. No CNVs in the FSTL5 gene, including small deletions/insertions, or other pathologic CNVs were detectable by Affymetrix array analysis in both these subjects; moreover, FSTL5 sequencing did not evidence any potentially damaging point mutation, in addition to the c.1358 $>$ C missense mutation.

The c.1358T > C transition leading to the p.Met453Thr substitution is reported in the dbSNP database (http://www.ncbi.nlm.nih.gov/snp/) as rs141999351 single nucleotide variant (SNV), with a global minor allele frequency of 0.0006 . None homozygous genotype for this variant is present in the 1000 Human Genome (Phase 3 release, $n=2504$ individuals; http://browser.1000genomes.org) as well as in the Exome Variant Server (release ESP6500SI-V2, $n=6503$ individuals; http://evs.gs.washington.edu/EVS/) databases.

Given its rarity, the identification of the rs141999351 missense variant in a patient who is de facto hemizygous for the FSTL5 gene suggests that it could contribute to the development of the disorder, acting through a recessive effect.

The FSTL5 gene is one of the five members (FSTL1-5) of the follistatin-like gene family, whose common feature is to encode for secreted extracellular glycoproteins with partial homology to follistatin, a well-known modulator of activin and other transforming growth factor- $\beta$ (TGF- $\beta$ ) superfamily members (Welt et al., 2002). So far, the functional role of FSTL5 has been poorly characterized. The high level of expression in the spinal cord tissue of early embryonic mice suggested its role in the development of the neurodorsal spinal cord and/or in the axonal guidance of dorsal root ganglion (Masuda et al., 2009). However, as retrieved from the Atlas database (http://www.ebi.ac.uk/gxa), FSTL5 expression is not limited to the spinal cord and it has been observed in different areas of the developing and adult central nervous system. These findings indicate that FSTL5 plays relevant roles in the organization and development of the brain, likely modulating the activity of TGF- $\beta$ superfamily members. Interestingly, a dysregulation in TGF- $\beta$ signaling pathway has been repetitively observed in schizophrenia. In particular, postmortem studies have shown an up-regulation of the TGF- $\beta$ signaling cascade in the hippocampus (Benes et al., 2007) and in the pyramidal neurons of schizophrenia subjects (Pietersen et al., 2014).

In conclusion, although functional studies would be required, the co-occurrence in the same patient with schizophrenia of two rare alterations in the FSTL5 gene (the rs141999351/p.Met453Thr missense variant and a CNV deleting almost the whole gene) suggests that these two mutational events may be relevant for the development of the disorder and indicates a possible role of FSTL5 in the development of schizophrenia.

\section{Conflict of interest}

The authors declare no conflicts of interest.

\section{Funding}

The study was supported by the Health Authority of the Lombardia Region (Project 153) together with grants from the Center of Behavioral and Neurodegenerative Disorders. These funding sources had no involvement in the study design, the collection, analysis, and interpretation of data, the 
writing of the report and the decision to submit the paper for publication.

\section{References}

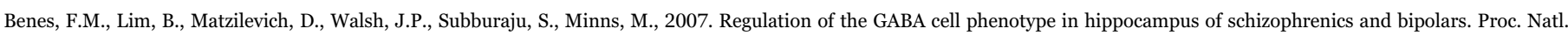
Acad. Sci. USA 104, 10164-10169. http://dx.doi.org/10.1073/pnas.0703806104.

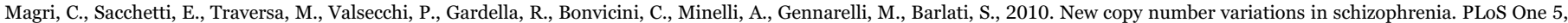
3-8. http://dx.doi.org/10.1371/journal.pone.0013422.

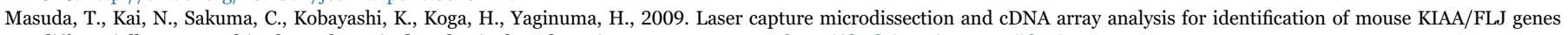
differentially expressed in the embryonic dorsal spinal cord. Brain Res. 1249, 61-67. http://dx.doi.org/10.1016/j.brainres.2008.10.028.

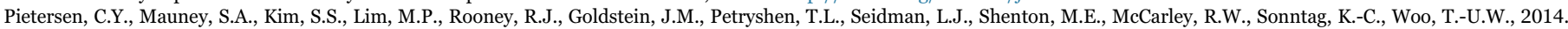
Molecular profiles of pyramidal neurons in the superior temporal cortex in schizophrenia. J. Neurogenet. 28, 53-69. http://dx.doi.org/10.3109/01677063.2014.882918.

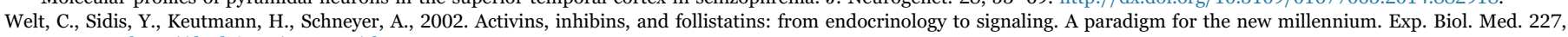
724-752. http://dx.doi.org/10.1258/ebm.2011.010287.

Rita Gardella', Emilio Sacchetti', Andrea Legati', Chiara Magri, Michele Traversa, Massimo Gennarelli'

Department of Molecular and Translational Medicine, Division of Biology and Genetics, University of Brescia, Viale Europa 11, 25123 Brescia,

Italy

Department of Clinical and Experimental Sciences, Neuroscience Section, University of Brescia, Viale Europa 11, 25123 Brescia, Italy

Department of Mental Health, Spedali Civili Hospital, P.le Spedali Civili 1, 25123 Brescia, Italy Genetics Unit, IRCCS San Giovanni di Dio, Fatebenefratelli, Via Pilastroni 4, 25125 Brescia, Italy

E-mail address: rita.gardella@unibs.it

\footnotetext{
* Corresponding author.
}

\footnotetext{
${ }^{1}$ Present address: Unit of Molecular Neurogenetics, The Foundation "Carlo Besta” Neurological Institute (IRCCS), Via Temolo 4, 20126 Milan, Italy.
} 